\title{
A Temperature Compensation Model for Low Cost Quartz Accelerometers and Its Application in Tilt Sensing
}

\author{
Weibin Yang, ${ }^{1}$ Bin Fang, ${ }^{2}$ Yuan Yan Tang, ${ }^{3}$ and Xudong Qin ${ }^{4}$ \\ ${ }^{1}$ College of Automation, Chongqing University, Chongqing 400044, China \\ ${ }^{2}$ College of Computer Science, Chongqing University, Chongqing 400044, China \\ ${ }^{3}$ Department of Computer and Information Science, University of Macau, Macau \\ ${ }^{4}$ Zhengzhou Horizon Electronic Science and Technology Co. Ltd., Zhengzhou 450000, China
}

Correspondence should be addressed to Bin Fang; fb@cqu.edu.cn

Received 23 May 2016; Revised 14 July 2016; Accepted 1 August 2016

Academic Editor: Michael Vynnycky

Copyright (C) 2016 Weibin Yang et al. This is an open access article distributed under the Creative Commons Attribution License, which permits unrestricted use, distribution, and reproduction in any medium, provided the original work is properly cited.

\begin{abstract}
Although the quartz accelerometer has made great advances, the performance, in some specific applications such as tilt sensing, needs to be well compensated in high temperature environment. Based on the high temperature testing of low cost quartz accelerometers, we found that the normalized positive and negative parts are asymmetrical at high temperature and the temperature curve of zero sensor output is related to the roll angle of the sensor. Traditional temperature compensation method only considers the temperature factor and ignores the roll sensitivity, which leads to deteriorated accuracy. To solve this problem, this paper proposes a novel and simple mathematical model to obtain a more accurate expression of zero sensor output, which makes the sensor output more robust at high temperature. Experimental results on two low cost quartz accelerometers demonstrate that the proposed model is feasible and effective, which could reduce the temperature drift error of the sensor output typically from $0.01 \mathrm{~g}$ to $0.001 \mathrm{~g}$. Furthermore, we introduce the compensated sensors in the three-axis inclinometer system for tilt sensing, and the evaluation results show that the temperature drift error of the inclination in the range $\left(20^{\circ} \mathrm{C}, 150^{\circ} \mathrm{C}\right)$ is reduced typically from $0.4^{\circ}$ to $0.1^{\circ}$ compared to the traditional method.
\end{abstract}

\section{Introduction}

Quartz accelerometers have become popular in many applications like motion control [1] and inertial measurement units [2] due to their simple construction and high accuracy. However, along with the increasing requirement for excellent performance of sensors, especially in harsh environment, for example, high temperature, high shock, and high vibration, some recalibration or recompensation methods should be adopted to obtain a satisfying accuracy depending on the specific usage $[3,4]$. Generally, the accuracy of the accelerometer can be influenced by manufacturing technique, internal structure, and surrounding environment in which the environmental temperature makes the performance much worse. A directed compensation method is to add a temperature control system [5], which could make the sensor work under the controlled temperature. However, the main drawbacks of the temperature control system include long stabilization time, large power, and increasing size, which cannot meet the actual demand in many applications. A commonly used alternative solution is to construct a mathematical model [6], which first models how the performance changes with temperature and then, according to the measured temperature of the sensor, corrects the sensor output. Apparently, such mathematical models are more suitable for most applications due to their simplicity and practicality.

Estimation methods need to be settled for the accelerometer in the thermal calibration in which iterative methods are mostly utilized to solve the calibration parameters (e.g., scale factors, misalignments, and biases of the accelerometer triad) and to achieve high estimation accuracy. Qian et al. [7] used least squares method to estimate the parameters of the proposed linear model. Yang et al. [8] presented an improved iterative nonlinear calibration method by using least squares method and sequence quadric program method. Ang et al. [9] proposed a nonlinear regression model to 
reduce the deterministic errors associated with scale factor, bias, and misalignment of the dual axis accelerometer, and the performance was verified by tilt and motion sensing. Won and Golnaraghi [10] proposed a mathematical model of six calibration parameters and use an iterative method to estimate the parameters of the nonlinear model. The objective of the proposed iterative method is to make the calculated gain factors and biases of each axis match the corresponding true values. Because the need of an initial rough estimate makes this algorithm inconvenient, Zhang et al. [11] proposed an improved multiposition calibration for solving the unknown parameters without any initial guess. However, the accelerometer temperature drift is still left as an unresolved problem. As a consequence, many trials have been made to establish thermal models of calibration parameters to attack this problem. Aggarwal et al. [12] explored the effects of thermal variations on biases and scale factors at different temperature values through the thermal chamber and then proposed three-order polynomial thermal models for ADI microelectromechanical system sensors. To investigate the thermal property in varying temperature conditions, Aggarwal et al. [13] considered the thermal ramp experiment from which a simple polynomial temperature model is developed for the inertial sensor biases and scale factors. After compensating the thermal errors, the inertial navigation solution was significantly improved. These two trials have to utilize a turntable with a temperature-controlled incubator that requires the precise orientation information. To improve the robustness to the turntable error, in [14], an indirect calibration technique for estimating the body-frame drift induced by the variation of the accelerometer sensitivity axes due to temperature changes is proposed. However, this estimation method depends on and is limited to the accuracy of horizontal accelerometer measurements. Moreover, Zhang et al. [15] utilized a nonvertical rotation axis observation method to attack the problem of the relationship between the gyroscope triad and the accelerometer triad in constant thermal conditions.

In this paper, we mainly focus on the usage of the quartz accelerometer in tilt sensing systems [16], such as Measurement While Drilling (MWD) and Logging While Drilling (LWD), which are used to monitor and guide the down borehole directional drilling in oil and gas exploration. Two main involved angles, inclination and roll angle, are illustrated in Figure 1. Suppose that an accelerometer is mounted and its sensitive axes are arranged along the $z$ axis in the $x y z$ coordinate system, inclination $\theta_{\text {inc }}$ is the angle that $z$-axis makes with the down direction $g$. It is $0^{\circ}$ when $z$-axis is down and $90^{\circ}$ when $z$-axis is horizontal. Roll angle $\theta_{\text {roll }}$ is defined as the angle of counterclockwise rotation about the $z$-axis (looking in the positive $z$-axis direction) in the gravitational field. Particularly, the inclination plays a very important role in tilt sensing systems, and the precision of inclination directly determines the performance of the whole system, so the accuracy of the sensor output is the key for accurate measurement at high temperature. In this paper, we pay our attention to the temperature compensation of low cost quartz accelerometers, and the main contributions include (1) proposing a mathematical temperature

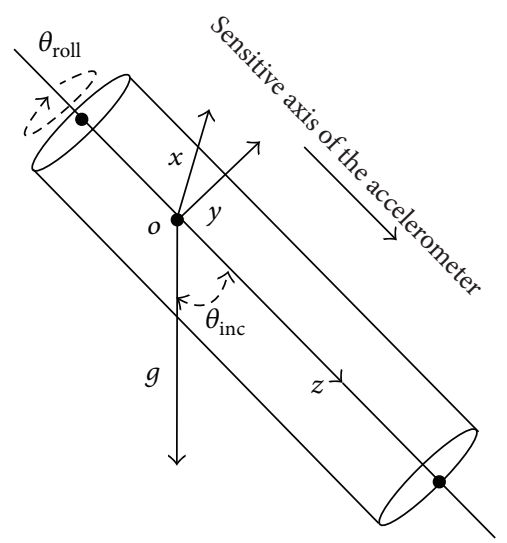

FIGURE 1: Definition of inclination and roll angle for tilt sensing. The sensitive axis of the tested accelerometer is along the $z$-axis.

compensation model for zero sensor output, especially, when the temperature curve changes with the roll angle, (2) presenting a feasible and effective solution for the whole compensation of quartz accelerometer, and (3) applying the proposed temperature compensation method in the tilt sensing system.

This paper is organized as follows: Section 2 contains the problem statement derived from our collected sensor data; Section 3 gives the description of the proposed temperature compensation method; Section 4 illustrates the experimental results and introduces the proposed model into the application of tilt sensing. Discussion and conclusions are drawn in Section 5.

\section{Problem Statement}

Although the performance of the quartz accelerometer is quite good to satisfy many applications at room temperature or under normal temperature, the accuracy may become poor and even unacceptable for some specific usage in high temperature environment. Generally speaking, temperature compensation is needed in those cases. However, we found that some characteristics of the sensor (e.g., asymmetry and roll sensitivity) may make the commonly used temperature compensation methods less effective.

To research how the performance changes with temperature, we have analyzed some sensor data from several quartz accelerometers. We use the monoaxial quartz accelerometer JSD-II/B-MJ, provided by the manufacturer (http://langfang037637.11467.com/), to constitute the hardware platform. Since the datasheet of this kind of accelerometer is not available online now, some of the most significant parameters are reported in Table 1 (although the provided measurement range is $\pm 10 \mathrm{~g}$, in our experiments, we only care about the static range of $\pm 1 \mathrm{~g}$ since the derived calibration model is only valid for this range, so the designed circuit only transfers the corresponding signals in the range $[-1.2 \mathrm{~g},+1.2 \mathrm{~g}])$. To compensate the sensor's temperature performance, the repeatability is the most important point. Fortunately, this basic assumption is still tenable in our 


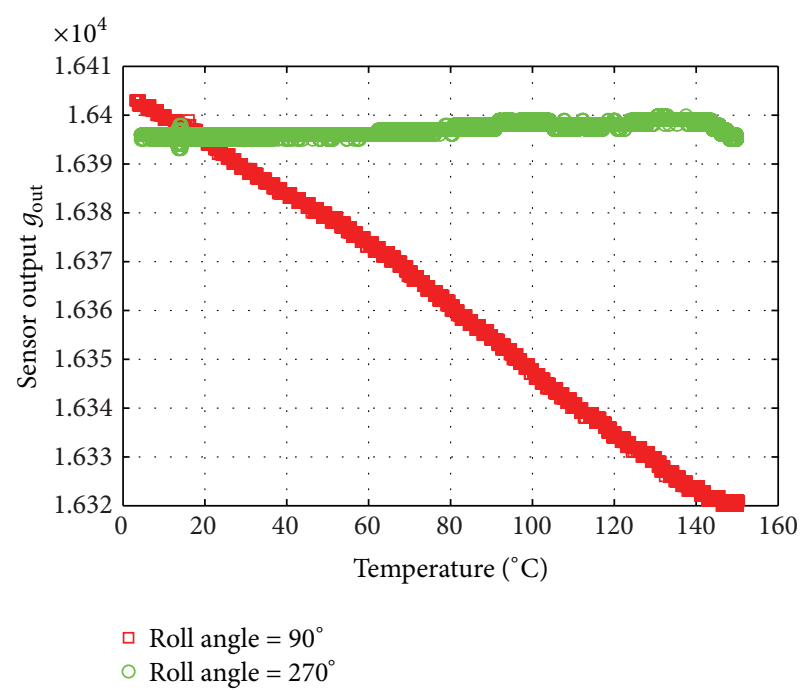

(a) Sensor \#1

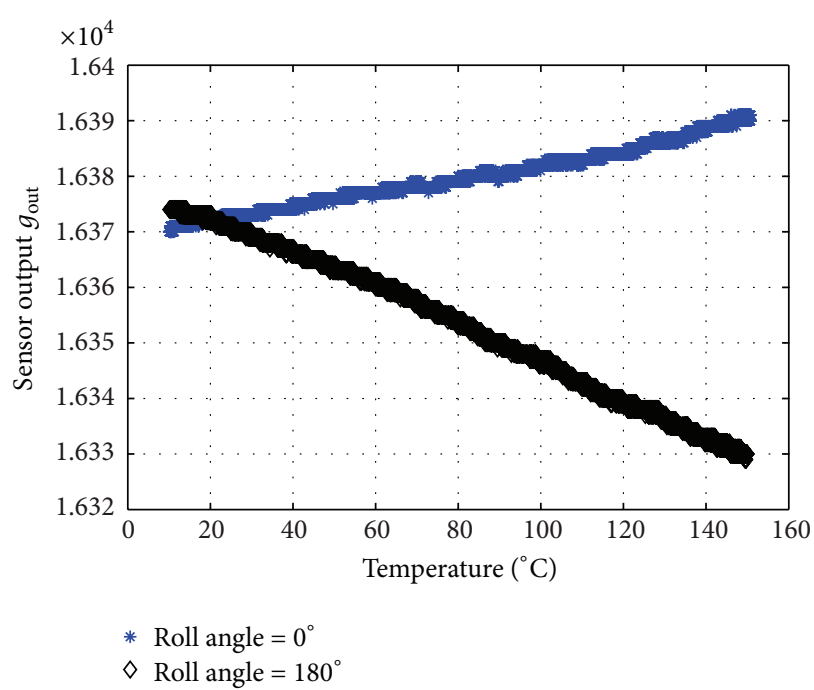

(b) Sensor \#2

FIGURE 2: Zero sensor output changes differently with temperature at different roll angles. Here $\theta_{\text {inc }}$ is close to $90^{\circ}$ and $g_{\text {out }}=5000 \cdot g_{\text {volt }}+16384$ is rescaled and digitalized from the output voltage $g_{\text {volt }}$. One number of $g_{\text {out }}$ corresponds to $0.2 \mathrm{mV}$ of the output voltage.

TABLE 1: Some of the most significant parameters of the monaxial quartz accelerometer. SF: scale factor. ppm: parts per million.

\begin{tabular}{lcc}
\hline Parameters & Value & Unit \\
\hline Operating temp. range & $-40 \sim+150$ & ${ }^{\circ} \mathrm{C}$ \\
Full scale range & \pm 10 & $\mathrm{~g}$ \\
Resolution/threshold $(@ 1 \mathrm{~Hz})$ & 0.05 & $\mathrm{mg}$ \\
Bias & \pm 10 & $\mathrm{mg}$ \\
Bias temp. coefficient & \pm 0.1 & $\mathrm{mg} /{ }^{\circ} \mathrm{C}$ \\
SF sensitivity & $1000 \pm 8$ & $\mathrm{mV} / \mathrm{g}$ \\
SF temp. coefficient & \pm 0.1 & $\mathrm{ppm} /{ }^{\circ} \mathrm{C}$ \\
\hline
\end{tabular}

observations. Meanwhile, we found that the data from one sensor are identical and repeatable, and the changing trend of the data is also consistent for different sensors. In our experiments, the maximum and minimum of the sensor output both change consistently with temperature when rotating the sensor to different roll angles, but the value of the zero sensor output changes differently with temperature at different roll angles (from Figure 1, when $\theta_{\text {inc }}=90^{\circ}$, the sensor output is close to zero; when $\theta_{\text {inc }}=0^{\circ}$, the sensor output is close to maximum; when $\theta_{\text {inc }}=180^{\circ}$, the sensor output is close to minimum. So we call them zero, maximum, and minimum sensor output, resp. To distinguish the term "bias," we use "zero sensor output" to express the approximate value when $\theta_{\text {inc }} \approx 90^{\circ}$ ). Note that, in the thermal chamber, only a mechnical fixture is used for fixing the cylinder structure in which the sensor is mounted, so it is hard to make $\theta_{\text {inc }}$ of the accelerometer accurately $90^{\circ}$. We assume that the trend of the temperature curve at near $90^{\circ}$ inclination is similar to that at precise $90^{\circ}$ inclination. For simplicity, when the inclination of the sensor is close to $90^{\circ}$, we also call it zero sensor output.
Some representative illustrations are shown in Figure 2. For instance, when the roll angle is $90^{\circ}$ and the inclination is near $90^{\circ}$, the sensor output of sensor $\# 1$ changes with the rising temperature while keeping the sensor static, and the data points are plotted with red squares in Figure 2(a). However, when we turn over the sensor to make the roll angle $270^{\circ}$ and the inclination is still near $90^{\circ}$, the change of the sensor output of sensor \#1 with the rising temperature becomes different, which is shown by green circles in Figure 2(a). The same situation occurs when the roll angles are $0^{\circ}$ and $180^{\circ}$ for sensor \#2, and the corresponding data are depicted in Figure 2(b). In the experiments, roll angles are roughly read out by a simple scaled circle, which is attached to the cylinder structure, since we found that the accuracy of roll angles has less influence on the accuracy of our model. To convert floating-point arithmetic to faster integer arithmetic in numerical calculation, the analog output voltage is first converted to digital signal by an $\mathrm{A} / \mathrm{D}$ (analog to digital) converter and then transformed by the formula $g_{\text {out }}=$ $5000 \cdot g_{\text {volt }}+16384$, where $g_{\text {volt }}$ is the digital output voltage (unit: V). In all our experiments, we use the digitalized $g_{\text {out }}$ as the sensor output. The phenomenon implies that the roll angle is relevant to the change of zero sensor output with rising temperature. All external environmental settings are identical except the change of the roll angle. It is worth noting that the phenomenon in Figure 2 should not be caused by random noises, for the differences of the zero sensor output at different roll angles could not be ignored and the phenomenon is regular and repeatable. In this paper, we only discuss the observed situation and find out how to compensate the data drift caused by temperature. Therefore, we adopt a data-driven method, by assuming that all possible errors, including the misalignment, temperature drift, and some uncertain errors caused by the operating environment, are compensated together. From Figure 2, since zero sensor 


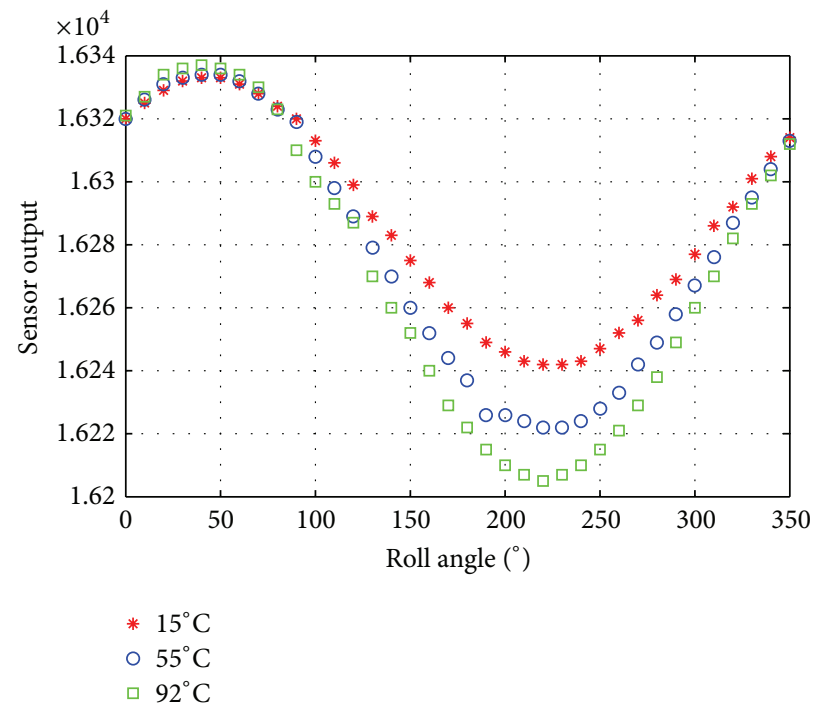

(a) Sensor output versus roll angle

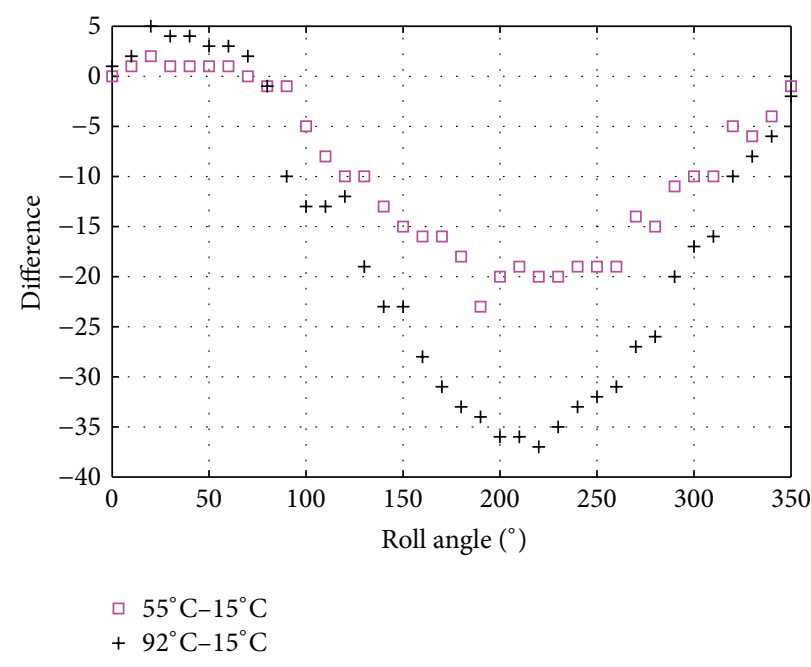

(b) Difference of sensor output versus roll angle

Figure 3: Sensor output changes with roll angle, and the difference of sensor output is an approximate sine function to roll angle. The sensor output and the difference in $y$-axis are both digitalized as in Figure 2, where one number of $g_{\text {out }}$ corresponds to $0.2 \mathrm{mV}$ of the output voltage.

output is approximately linear with temperature at any roll angle, for simplicity, we can fit it using a linear function. As a result, we give an assumption as follows.

Assumption 1. The zero sensor output is approximately linear with temperature at any roll angle, but the slope may change with roll angles.

Moreover, we further investigate the relationship between the sensor output and three possible factors: roll angle, temperature, and inclination. For an ideal situation, the sensor output should satisfy $g_{\text {out }}=g \cdot \cos \theta_{\text {inc }}$. But it is impossible because our purpose is to obtain an accurate inclination $\theta_{\text {inc }}$ by using the sensor output. Since temperature curve of the sensor output is related to the roll angle, we try to utilize the roll angle to compensate the temperature drift of the sensor output. Therefore, when the inclination is fixed in the thermal chamber, we record the sensor output of the tested accelerometer at every roll angle with $10^{\circ}$ interval in the range $\left[0^{\circ}, 360^{\circ}\right]$ at three stable temperature points (to avoid the temperature hysteresis, all experimental data are recorded after staying at least 20 minutes at an expected temperature point). As a result, as shown in Figure 3(a), at a fixed inclination and a fixed temperature, the relation between the sensor output and roll angle is an approximate sine function because of the misalignment of the rotation axis and the sensitivity axis of the accelerometer. Hence the plots in Figure 3(a) are the projection of the rotation into the $\theta_{\text {roll }}$ and $g_{\text {out }}$ plane and is hence describing a sine curve with an offset. When the temperature changes, for example, from $15^{\circ} \mathrm{C}$ to $52^{\circ} \mathrm{C}$ and then $92^{\circ} \mathrm{C}$, the sine curves become different. As a result, as illustrated in Figure 3(b), the difference of the sensor output at different temperature can be also approximated by a sine function of roll angle. The magenta squares depict the differences of sensor output between temperature points $52^{\circ} \mathrm{C}$ and $15^{\circ} \mathrm{C}$, and the black crosses stand for that between $92^{\circ} \mathrm{C}$ and $52^{\circ} \mathrm{C}$. Furthermore, we found that the amplitude of the sine curve in Figure $3(\mathrm{~b})$ is related to the inclination, and the function could be also an approximate sine curve. Due to the limited experiment condition, we cannot give out a lot of points at different inclination angles to fit the sine curve. We tested some specific positions to give a rough estimation. For instance, the maximum amplitude of the sine curve at $90^{\circ}$ inclination is 37 , which can be expressed as $37=37 * \sin 90^{\circ}$, while the maximum amplitude of the sine curve at $50^{\circ}$ inclination is about 27 , which can be represented as $27 \approx 37 * \sin 50^{\circ}$. In fact, as it will be mentioned in Section 3.2, we only care about the zero sensor output, so this assumption is not necessary for our proposed model. However, we could provide the assumption in case that some possible applications need it. And also it makes 90。 inclination a specific example and easy to extend. In our model, it is beneficial to know that the maximum amplitude of the sine curve in Figure 3(b) is also an approximate sine function to inclination when the inclination is $90^{\circ}$. Therefore, we give another assumption below.

Assumption 2. The difference of sensor output under different temperature could be approximate sine functions to roll angle, and the amplitude of the sine curve is also an approximate sine function to inclination.

\section{Temperature Compensation Method}

Inspired by abovementioned observations, we propose a novel temperature compensation procedure, which can simultaneously reduce the negative effect brought by temperature and roll angle. The proposed method is composed of the following four steps. 
3.1. Modeling Temperature Curves of Maximum and Minimum. The bias and scale factor may suffer deteriorated performance when the accelerometer is used in high temperature and high accuracy required measurement system, probably because of different nonlinear temperature drift of the complex peripheral interface circuit. Therefore, for better expressing the temperature drift, we adopt a polynomial model here to obtain the temperature curve of maximum and minimum output of the sensor, which can be written as

$$
g_{m}=\sum_{i=0}^{n} \alpha_{i} \cdot t^{i}
$$

where $g_{m}$ is the maximum or minimum output, $\alpha_{i}$ is the corresponding nonlinear coefficient, and $t$ is the temperature. $n$ is empirically set to 4 in our system due to the tradeoff between the effective repeatability of the maximum and minimum output and overfitting. In fact, the sensor is not sensitive at the position of the maximum or minimum output, so it is easy to find the proper position for maximum or minimum output in the actual operation (for convenience, the compensated accelerometer is mounted into a cylinder structure, and the direction of the maximum sensor output is the same with the gravity).

3.2. Modeling Temperature Curve of Zero Sensor Output. The key step, also the significant difference of our proposed procedure, is to obtain the temperature curve of the zero sensor output. Due to the manufacturing technique or internal structure, the temperature curve of the zero sensor output changes with the roll angle at a fixed inclination, which makes the usual temperature compensation method less effective. The direct result is that the normalized positive and negative parts are asymmetrical at high temperature. Formally, that is, $\left(g_{\text {out }}-g_{0}\right) /\left(g_{\text {max }}-g_{0}\right) \neq\left(g_{\text {out }}-g_{0}\right) /\left(g_{0}-g_{\text {min }}\right)$, where $g_{\text {out }}$ is the actual output of the sensor and $g_{\text {max }}, g_{\text {min }}$, and $g_{0}$ are maximum, minimum, and zero sensor output, respectively. To handle this problem, we need to obtain a more accurate temperature curve of zero sensor output.

Assume that the function between the zero sensor output and the temperature is linear (Assumption 1), which could be defined as

$$
y=k \cdot t+b
$$

where $k$ is the slope and $b$ is the offset. Suppose that two points $\left(t_{1}, y_{1}\right)$ and $\left(t_{2}, y_{2}\right)$ can determine this linear function, in which $\left(t_{1}, y_{1}\right)$ is a point at room temperature $t_{1}$, so $k$ could be calculated by $k=\left(y_{2}-y_{1}\right) /\left(t_{2}-t_{1}\right)$ and $b$ could be $b=y_{1}-k \cdot t_{1}$. According to Assumption 2, if we set $\Delta y=y_{2}-y_{1}$, then $\Delta y$ could satisfy the function

$$
\begin{aligned}
\Delta y & =f\left(\theta_{\text {inc }}, \theta_{\text {roll }}\right) \\
& =\sin \theta_{\text {inc }} \cdot\left(A \cdot \sin \left(\omega \cdot \theta_{\text {roll }}+\varphi\right)+h\right),
\end{aligned}
$$

where $A$ is the amplitude, $\omega \cdot \theta_{\text {roll }}+\varphi$ is the phase, and $h$ is the offset. In addition, since we only need to obtain the temperature curve of zero sensor output, the inclination is always about $90^{\circ}$; that is, $\theta_{\text {inc }} \approx 90^{\circ}$. Here we assume that $\theta_{\text {inc }}=90^{\circ}$, and we experimentally found that this assumption has no obvious influence on the whole compensation procedure. $\omega$ is set to 1 in our model, and it is reasonable to consider it as a complete sine period when the sensor turns over from roll angle $0^{\circ}$ to $360^{\circ}$. The experimental data in Figure 3 could also prove that. Therefore, (3) could be simplified as

$$
\Delta y=f\left(\theta_{\text {roll }}\right)=A \cdot \sin \left(\theta_{\text {roll }}+\varphi\right)+h .
$$

Therefore, as long as two points $\left(t_{1}, y_{1}\right)$ and $\left(t_{2}, y_{2}\right)$ are obtained, then $k=\Delta y /\left(t_{2}-t_{1}\right)$ and $b=y_{1}-\left(\Delta y /\left(t_{2}-t_{1}\right)\right) \cdot t_{1}$. Substitute $k, b$, and (4) into (2); then temperature compensation function of zero sensor output could be represented by

$$
\begin{aligned}
y= & F\left(\theta_{\text {roll }}, t\right) \\
= & \frac{A \cdot \sin \left(\theta_{\text {roll }}+\varphi\right)+h}{t_{2}-t_{1}} \cdot t \\
& +\left(y_{1}-\frac{A \cdot \sin \left(\theta_{\text {roll }}+\varphi\right)+h}{t_{2}-t_{1}} \cdot t_{1}\right) .
\end{aligned}
$$

3.3. Solving Model Parameters. Since we have obtained three expressions of maximum, minimum, and zero sensor output changing with temperature, we need to get all involved parameters, that is, $\alpha_{i}$ in (1) and $A, \varphi$, and $h$ in (4). The temperature $t$ is required from a temperature sensor, and the roll angle $\theta_{\text {roll }}$ could be controlled manually in laboratory tests or approximately calculated by another two accelerometers in the three-axis tilt sensing system. For simplicity, we adopt the nonlinear least squares regression method [17] to optimize these parameters by collecting some necessary data. To obtain the coefficient estimates, the least squares method minimizes the summed square of residuals. The residual for the $i$ th data point $r_{i}$ is defined as the difference between the observed response value $y_{i}$ and the fitted response value $\hat{y}_{i}$ and is identified as the error associated with the data; that is, $r_{i}=$ $y_{i}-\hat{y}_{i}$. The summed square of residuals is given by

$$
S=\sum_{i=1}^{n} r_{i}^{2}=\sum_{i=1}^{n}\left(y_{i}-\widehat{y}_{i}\right)^{2}
$$

where $n$ is the number of data points included in the fit and $S$ is the sum of squares error estimate. For example, (5) can be rewritten as $y=F(X, \beta)+\varepsilon$, where $y$ is an $n$-by- 1 vector of responses, $f$ is a function of $\beta$ and $X, \beta$ is an $m$-by- 1 vector of coefficients, $X$ is the $n$-by- $m$ design matrix for the model, $\varepsilon$ is an $n$-by- 1 vector of errors, $m$ is the number of the parameter, and $n$ is the number of data points. We choose trust-region fitting algorithm [17] in the iterative procedure until the fit reaches the specified convergence criteria.

3.4. Normalizing Sensor Output. Since the output of accelerometer is often considered as symmetrical even at high temperature, that is, $g_{0}=\left(g_{\max }+g_{\min }\right) / 2$, classical normalization method [18] is often defined as

$$
g_{n}=\frac{g_{\mathrm{out}}-g_{0}}{g_{\max }-g_{0}}=2 \frac{g_{\mathrm{out}}-g_{\mathrm{min}}}{g_{\max }-g_{\min }}-1,
$$


TABLE 2: Collected data points from two quartz accelerometers. The sensor output is digitalized as in Figure 2.

\begin{tabular}{ccccc}
\hline & Roll angle & $19.8^{\circ} \mathrm{C}$ & $146^{\circ} \mathrm{C}$ & Difference \\
\hline & $30^{\circ}$ & 16398 & 16402 & 4 \\
Sensor \#1 & $120^{\circ}$ & 16365 & 16338 & -27 \\
& $210^{\circ}$ & 16317 & 16245 & -72 \\
& $300^{\circ}$ & 16350 & 16310 & -40 \\
\hline \multirow{5}{*}{ Sensor \#2 } & Roll angle & $22.8^{\circ} \mathrm{C}$ & $147^{\circ} \mathrm{C}$ & Difference \\
& $30^{\circ}$ & 16330 & 16361 & 31 \\
& $120^{\circ}$ & 16402 & 16406 & 4 \\
& $210^{\circ}$ & 16392 & 16352 & -40 \\
& $300^{\circ}$ & 16321 & 16305 & -16 \\
\hline
\end{tabular}

where $g_{n}$ is the normalized sensor output. As mentioned above, due to the possible asymmetrical performance of low cost quartz accelerometer, classical normalization method may be not accurate, so that the usage is limited. Assuming that we have obtained a feasible temperature curve of zero sensor output, then the normalization of our model could be

$$
g_{n}= \begin{cases}\frac{g_{\text {out }}-g_{0}}{g_{\text {max }}-g_{0}} & g_{\text {out }} \geq g_{0} \\ \frac{g_{\text {out }}-g_{0}}{g_{0}-g_{\min }} & g_{\text {out }}<g_{0} .\end{cases}
$$

Since in static condition $g_{\max }$ should be equal to $1 \mathrm{~g}$ and $g_{\min }$ should be equal to $-1 \mathrm{~g}$, the unit of the normalized sensor output $g_{n}$ could be $g$. Note that our proposed normalization is in fact an approximation to a nonlinear characteristic of the accelerometer, which may not be strictly realistic. However, it is reasonable to make such an approximation based on the observations mentioned above which is also proved to be effective by the following experimental results.

\section{Experimental Results and Validation in Tilt Sensing}

In order to test the proposed temperature compensation method, we first make temperature compensation to two low cost quartz accelerometers and then introduce the model into the three-axis tilt sensing system.

4.1. Experimental Results in Sensors Compensation. First we place each sensor (mounted into a cylinder structure) at the maximum and minimum positions in the thermal chamber, respectively, and collect the sensor output when temperature rises from room temperature to the upper limit temperature, that is, $150^{\circ} \mathrm{C}$, and then these recorded data are used to solve the parameters in (1) by using the least squares regression method. We also collect some data points from two quartz accelerometers to obtain the parameters in (5), which is shown in Table 2. For simplicity, the data points should be as few as possible, because less data collection can simplify the production process. Besides, the data in our experiments are often recorded at high temperature, so less data points could also largely reduce the heating time, which is helpful for the experiments or production. In our experiments, we found that the readouts at 4 roll angles and 2 temperature points are enough to solve the parameters in (5). As shown in Table 2 , we choose $30^{\circ}, 120^{\circ}, 210^{\circ}$, and $300^{\circ}$ as 4 tested roll angles and a room temperature point (about $20^{\circ} \mathrm{C}$ ) and a high temperature point (close to $150^{\circ} \mathrm{C}$ ) as 2 tested temperature points. It is worth noting that any 4 roll angles increasing by about $90^{\circ}$ are acceptable for training the model. In addition, the accuracy of roll angles and temperatures are unnecessarily too high, because we found that these factors do not affect the result too much. In our actual operation, we first record 4 output values with 4 different roll angles at room temperature before heating, then heat up the accelerometers to the upper limit temperature $150^{\circ} \mathrm{C}$ under the heating rate $1^{\circ} \mathrm{C}$ per minute, and rotate the sensor to record another 4 values with corresponding 4 roll angles when the temperature is almost stable (after staying at least 20 minutes at $150^{\circ} \mathrm{C}$ ). The heating rate $1^{\circ} \mathrm{C}$ is set empirically by considering the operating environment of MWD or LWD systems. Besides, since the accelerometer cannot be very accurately mounted along the $z$-axis of the cylinder structure, the data points at room temperature are not identical, as shown in Table 2, which means that the inclination of the accelerometer is not accurately $90^{\circ}$ while recording the data points. However, assisted by a standard measurement apparatus, which can provide the indication of the inclination with an accuracy of about 0.01 , we found that at room temperature the zero sensor values at different roll angles are nearly equal. It means that the normalized positive and negative parts are symmetrical at room temperature, so the first point $\left(t_{1}, y_{1}\right)$ in (5) can be obtained by $y_{1}=\left(g_{\max }^{t_{1}}+g_{\min }^{t_{1}}\right) / 2$, where $g_{\max }^{t_{1}}$ and $g_{\min }^{t_{1}}$ are the maximum and minimum at room temperature $t_{1}$, respectively. In this experiment, the first point is $(19,16386)$ for sensor \#1 and $(21,16373)$ for sensor $\# 2$.

Utilizing the collected data, we solve the model parameters by the least squares regression method. As a consequence, the model parameters $(A, \varphi, h)$ of sensors $\# 1$ and $\# 2$ are $(38.55$, $0.8778,-33.75)$ and $(36.88,0.7726,-5.25)$, respectively. As shown in Figure 4, the proposed model could be fitted well. Since we have simplified the model, it is easy to determine the whole sine curve by using only 4 data points.

Once we have obtained the parameters in (1) and (5), we can get the temperature curves of the maximum, minimum, and zero sensor output, respectively, and then the normalized sensor output could be calculated. The comparison of the normalized sensor output by using (7) and (8) is illustrated in Figure 5, in which "classical" and "proposed" represent the results obtained by using (7) and (8), respectively. We randomly place the sensor at different roll angles where the inclination is close to $90^{\circ}$. Figure 5 shows some results when the roll angles are about $30^{\circ}, 180^{\circ}$, and $330^{\circ}$ for both sensors \#1 and \#2. We can see that our normalized sensor output changes much less than the classical method when the temperature rises from room temperature to even $150^{\circ} \mathrm{C}$. Comparing with the classical method, the proposed temperature method could reduce the temperature drift error typically from $0.01 \mathrm{~g}$ (at least $0.005 \mathrm{~g}$ ) to $0.001 \mathrm{~g}$ ( $\mathrm{g}$ is the unit of the normalized sensor output obtained by (8)), which demonstrates that our method is more stable and less sensitive to high temperature 


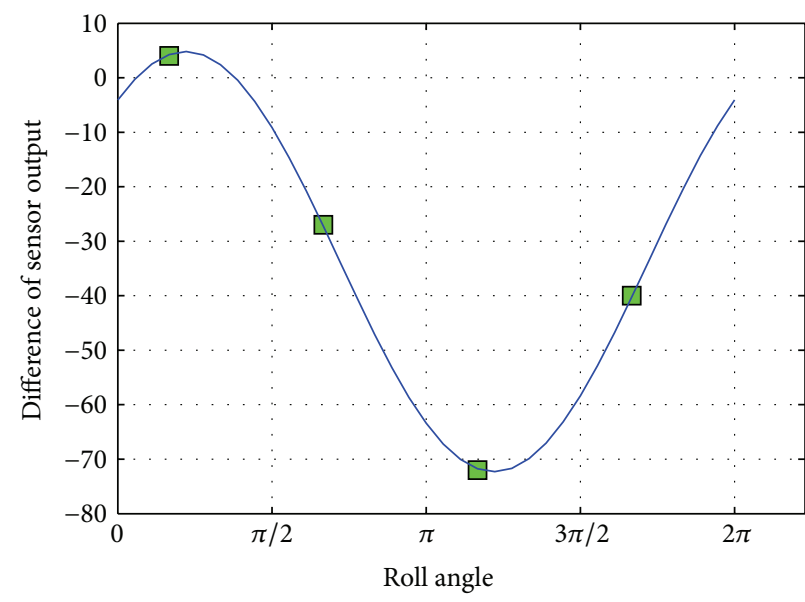

(a) Sensor \#1

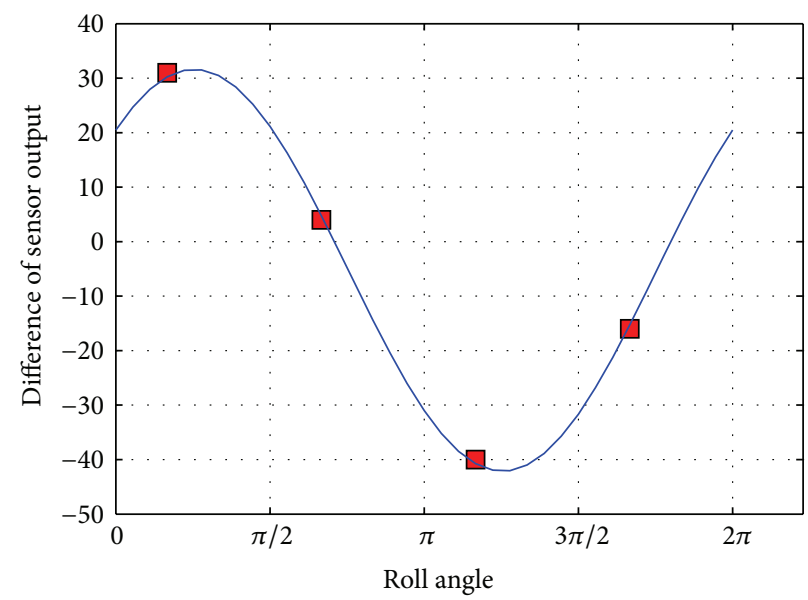

(b) Sensor \#2

Figure 4: The fitting results. (a) Sensor \#1. (b) Sensor \#2. The difference of sensor output in $y$-axis is digitalized as in Figure 2, where one number of $g_{\text {out }}$ corresponds to $0.2 \mathrm{mV}$ of the output voltage.

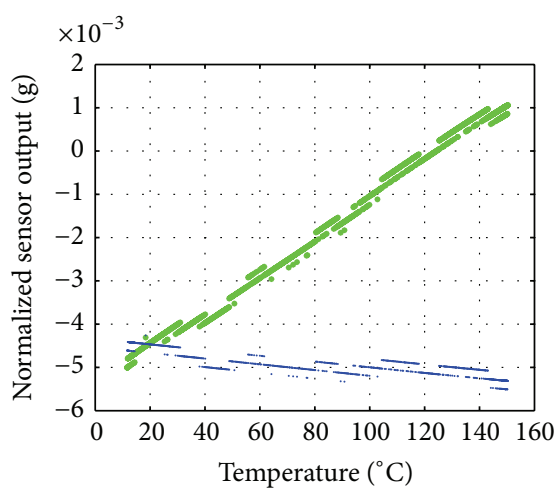

- Classical

Proposed

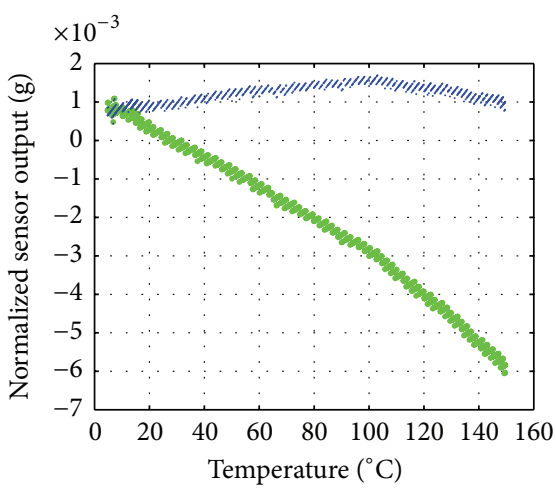

- Classical

Proposed

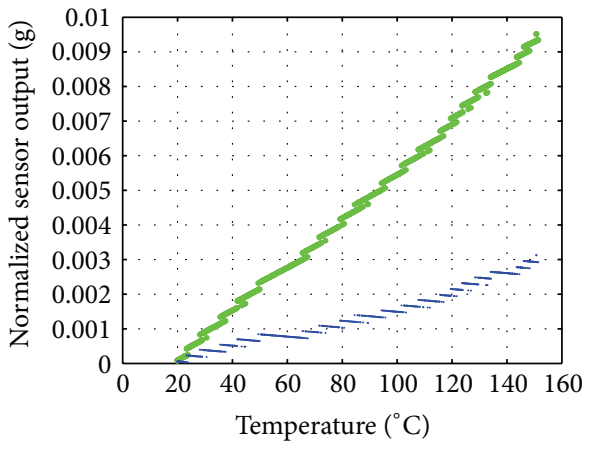

- Classical

Proposed

(a) Sensor \#1. The roll angles are about $30^{\circ}, 180^{\circ}$, and $330^{\circ}$ and the inclination is close to $90^{\circ}$

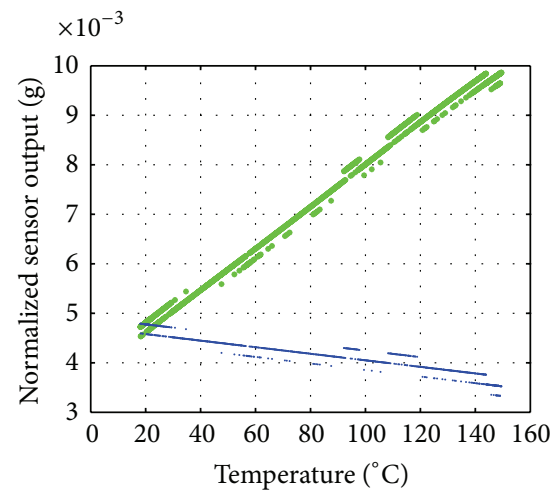

- Classical

Proposed

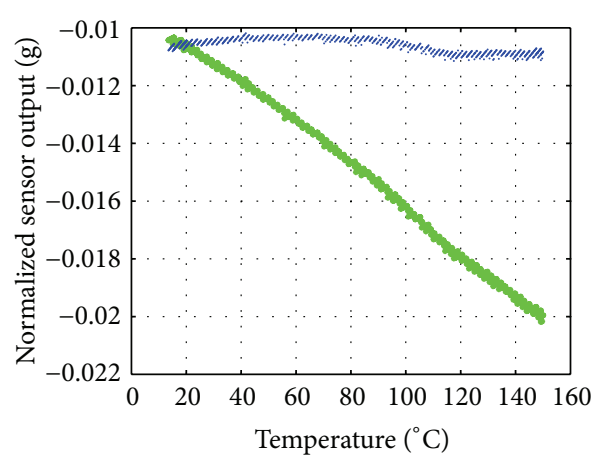

- Classical

Proposed

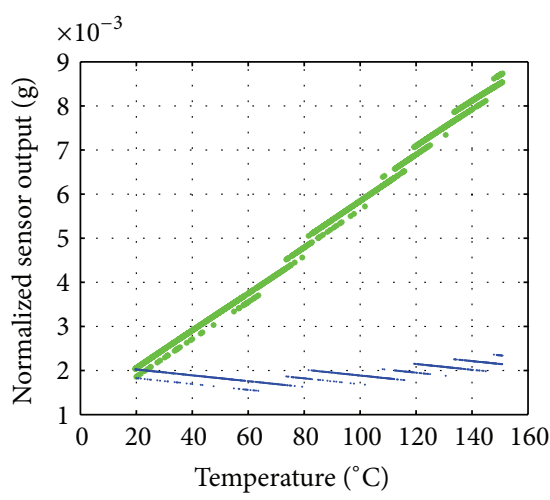

- Classical

Proposed

(b) Sensor \#2. The roll angles are about $30^{\circ}, 180^{\circ}$, and $330^{\circ}$ and the inclination is close to $90^{\circ}$

FIGURE 5: The comparison of the normalized sensor outputs between the proposed and the classical temperature compensation methods. 


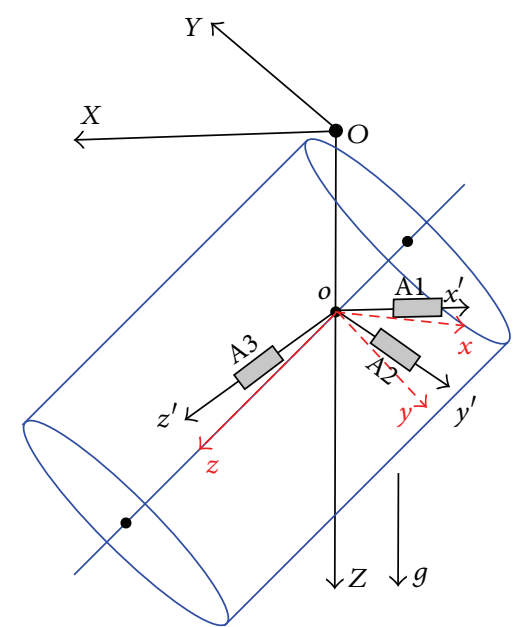

FIgURE 6: Sensor model for a cylindrical inclinometer [19].

environment. The compensated accelerometers could be easily adopted in tilt sensing systems in high temperature environment.

4.2. Experimental Validation in Tilt Sensing. To further verify the feasibility of the proposed temperature compensation method, we apply it to tilt sensing at high temperature. We comply with the basic assumption that an inertial sensor, in static condition, is subjected only to the gravity force. As a consequence, the module of the acceleration vector measured by the sensor has to be equal to $g$, independent of the sensor orientation [20]. As shown in Figure 6, in a 3D XYZ Cartesian coordinate system, $Z$ has the same direction with the gravity $g$, and $X O Y$ plane is perpendicular to $g$. In a cylindrical inclinometer instrument, three quartz accelerometers are mounted along three axes of $x y z$ coordinate system. Due to the limitation of the installation process, sensitive axes of accelerometers are often not accurately identical with the corresponding direction. This results in the misalignment. Usually, the misalignment should be calibrated to obtain a satisfying accuracy. Since in this paper we do not focus on the misalignment calibration algorithm, for simplicity, we use the model in [19] to obtain the final inclination. After the temperature compensation procedure, we denote the normalized actual accelerometer output as $\mathbf{G}^{\prime}=\left[g_{x^{\prime}}, g_{y^{\prime}}, g_{z^{\prime}}\right]^{T}$ (temperature compensation and normalization steps are introduced in Section 3) and the acceleration vector in the ideal $x y z$ coordinate system as $\mathbf{G}=\left[g_{x}, g_{y}, g_{z}\right]^{T}$.

The mathematical model describes the accelerometer output in matrix form as

$$
\mathbf{G}=\mathbf{S}\left(\mathbf{G}^{\prime}-\mathbf{O}_{\mathbf{g}}\right),
$$

where $\mathbf{S}$ is the $3 \times 3$ sensitivity matrix and $\mathbf{O}_{\mathbf{g}}=$ $\left[O_{g x}, O_{g y}, O_{g z}\right]^{T}$ is the offset. The diagonal elements of $\mathbf{S}$ represent the scale factors along the three axes, whereas the other elements of $\mathbf{S}$ are called cross-axis factors. These terms allow describing the axes misalignment, the cross-axis sensitivity, and the mechanical imperfection effect between different channels caused by the sensor electronics. Finally, the inclination angle could be computed by $[20,21]$

$$
\theta_{\mathrm{inc}}=\arctan \frac{\sqrt{g_{x}^{2}+g_{y}^{2}}}{g_{z}} .
$$

We test the proposed temperature compensation method on two inclinometer devices, which are noted as devices \#1 and \#2. A two-step procedure is adopted in our experiments, in which the temperature performance of each sensor is first compensated, and then a calibration method for three accelerometers is used for tilt sensing. We first make temperature compensation for each quartz accelerometer as mentioned in Section 3 and then place them in each inclinometer device as their own $x$-, $y$-, and $z$-axes. After obtaining the model parameters of each accelerometer, each compensated sensor output of $x, y$, and $z$ could be calculated, respectively, where the roll angle of each sensor could be estimated by another two axes; for example, for $z$-axis, the roll angle could be $\arctan \left(g_{y} / g_{x}\right)$. To evaluate the effect of our proposed temperature compensation method, we fix each device into a thermal chamber with different inclination angles (here we randomly choose $90^{\circ}, 50^{\circ}$, and $30^{\circ}$ for evaluation) and then heat it up from room temperature to $150^{\circ} \mathrm{C}$ (staying at least 20 minutes to keep the temperature at stable $150^{\circ} \mathrm{C}$ ) under the heating rate of $1^{\circ} \mathrm{C}$ per minute. The heating rate of $1^{\circ} \mathrm{C}$ is set empirically by considering the operating environment of MWD or LWD systems. We record all inclination angles with and without our proposed temperature compensation method during the heating process, and the experimental results are illustrated in Figure 7 . Inclination angles $(y$ axes) change as the temperature ( $x$-axes) rises from the room temperature to the upper limit temperature of $150^{\circ} \mathrm{C}$. Similarly, "classical" and "proposed" represent the results obtained by using classical normalized method (see (7)) and ours (see (8)), respectively. To distinguish the influence brought by temperature and other factors (e.g., scale factor, bias, and misalignment) in tilt sensing, both "classical" and "proposed" methods calibrate all parameters by the model in [19] except the temperature compensation coefficients, so that the differences of evaluation results are only caused by different temperature compensation methods. Typically the temperature drift error of the inclination calculated by our method is only about $0.1^{\circ}$, which is more robust than the drift error $0.4^{\circ}$ computed by using the classical temperature method. Furthermore, according to (10), when the inclination angle is near $90^{\circ}, g_{z}$ plays the most important role and $g_{x}$ and $g_{y}$ have little or even no effect on the tilt angle. In fact, we found in our experiments that at least up to $60^{\circ}$ we can only compensate $g_{z}$ by our new method and solve $g_{x}$ and $g_{y}$ using the classical compensation method for simplicity, and the results are very close.

\section{Conclusions}

This paper proposes a mathematical temperature compensation procedure for low cost quartz accelerometer based on the observation that zero sensor output changes with 

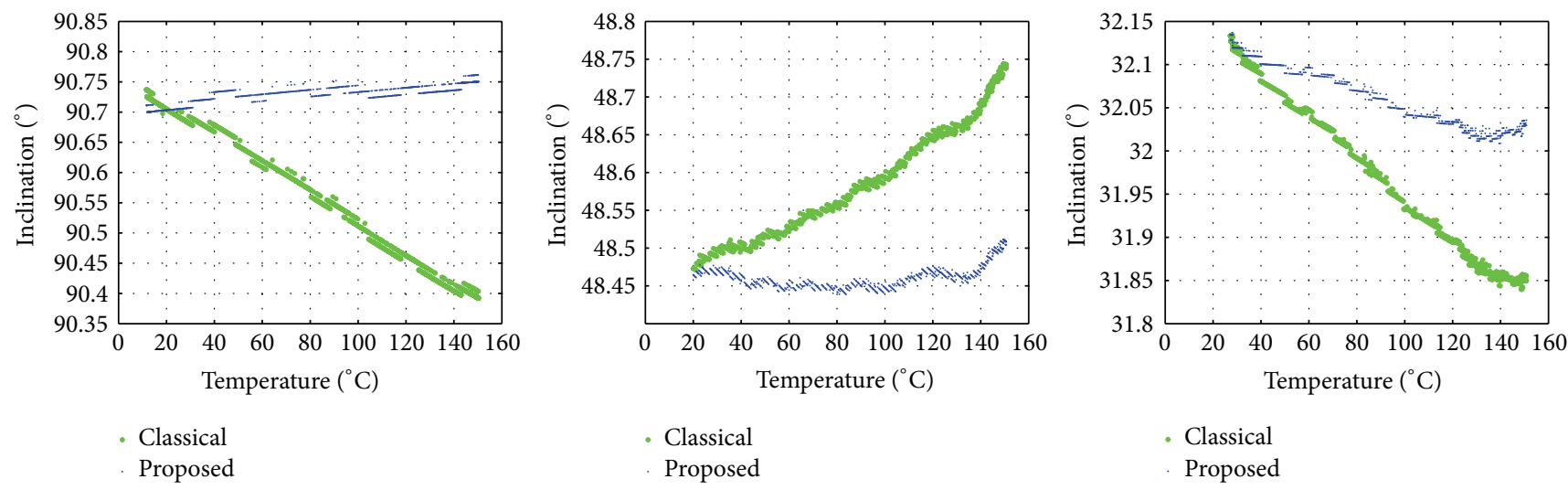

(a) Device \#1. The three-axis inclinometer is placed with $90^{\circ}, 50^{\circ}$, and $30^{\circ}$ inclination with arbitrary roll angles

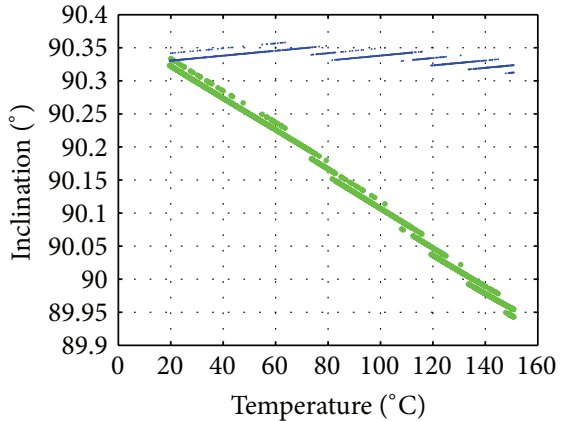

- Classical Proposed
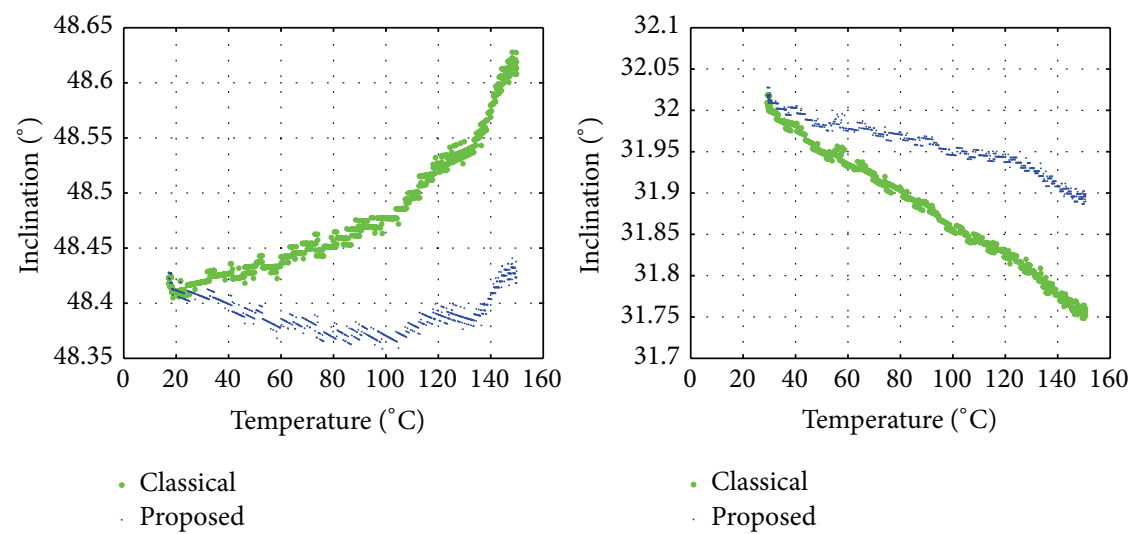

(b) Device \#2. The three-axis inclinometer is placed with $90^{\circ}, 50^{\circ}$, and $30^{\circ}$ inclination with arbitrary roll angles

FigurE 7: The comparison of the inclination drift errors between the proposed and the classical temperature compensation methods while heating up.

both roll angle and temperature. By modeling maximum, minimum, and zero sensor output, respectively, we solve the problem that the normalized positive and negative parts of sensor output under high temperature environment are asymmetrical. To make it easy to operate, we only choose several representative data points (responding to 4 roll angles at low and high temperature and a common point at room temperature) to fit the zero sensor output curve. Although the model is not very accurate because some approximations and assumptions are adopted, the experimental results demonstrate that it can distinctly reduce the temperature drift error of the sensor output. Moreover, we apply the proposed temperature compensation method in the tilt sensing system, which shows the actual efficiency at high temperature environment.

In addition, some points should be noticed. First, we ignore the long-term drifts of the tested accelerometers in our experiments because the duration of the experiments is short enough. Second, 8 accelerometers are tested in our experiments ( 2 in Section 4.1 and 6 in Section 4.2), and all the tested sensors have similar temperature characteristics. By using our proposed temperature compensation model, the temperature performances of all the tested sensors are greatly improved. Third, low cost quartz accelerometers are usually several times cheaper than well-produced ones; however, by using our proposed temperature compensation model, they can be also adopted for accurate tilt sensing even in harsh temperature environment. Fourth, it is important to obtain the data points accurately, for example, $\left(t_{1}, y_{1}\right)$ and $\left(t_{2}, y_{2}\right)$, for a small mistake in those readings can cause a significant change of the unknown parameters. Fortunately these values are quite easy to obtain in the process and should not be mistaken easily, because the temperature readout and the sensor output are the base of the whole measurement system. Meanwhile, if the data points are wrongly collected, a simple verification at high temperature would help. Finally, this paper gives some experimental data and proposes an effective compensation model to improve the temperature performance of the low cost quartz accelerometer, but, limited to the existing test condition, it is hard to compare with many types of low cost accelerometers to build a more general compensation model. The proposed mathematical model is a data-driven method essentially, which tries to compensate all possible errors (misalignment, temperature drift, etc.) of low cost accelerometers. Therefore, low cost quartz accelerometers with these similar characteristics could 
use our proposed model to measure tilt in static situation. Future work may lie in comparing with different types of accelerometers and proposing a more general model.

\section{Competing Interests}

The authors declare that there are no competing interests regarding the publication of this paper.

\section{Acknowledgments}

This research was supported in part by National Natural Science Foundation of China (61472053, 91420102), China Postdoctoral Science Foundation (2014M550456), Chongqing Postdoctoral Special Funding Project (Xm2014087), and Basic Science and Advanced Technology Research Project of Chongqing (cstc2015jcyjA40041).

\section{References}

[1] V. S. Lobanov, N. V. Tarasenko, D. N. Shulga, V. N. Zboroshenko, and V. P. Fedotov, "Fiber-optic gyros and quartz accelerometers for motion control," IEEE Aerospace and Electronic Systems Magazine, vol. 22, no. 4, pp. 23-29, 2007.

[2] J. Yang, W. Wu, Y. Wu, and J. Lian, "Thermal calibration for the accelerometer triad based on the sequential multiposition observation," IEEE Transactions on Instrumentation and Measurement, vol. 62, no. 2, pp. 467-482, 2013.

[3] J. Guo and M. Zhong, "Calibration and compensation of the scale factor errors in DTG POS," IEEE Transactions on Instrumentation and Measurement, vol. 62, no. 10, pp. 27842794, 2013.

[4] D. Xu, Y. Chen, and R. Kang, "Study of accelerated stability test method for quartz flexible accelerometer," IEEE Transactions on Device and Materials Reliability, vol. 11, no. 1, pp. 148-156, 2011.

[5] K. I. Lee, H. Takao, K. Sawada, and M. Ishida, "A threeaxis accelerometer for high temperatures with low temperature dependence using a constant temperature control of SOI piezoresistors," in Proceedings of the 16th IEEE International Conference on Micro Electro Mechanical Systems (MEMS '03), pp. 478-481, January 2003.

[6] Y. Pan, L. Li, C. Ren, and H. Luo, "Study on the compensation for a quartz accelerometer based on a wavelet neural network," Measurement Science and Technology, vol. 21, no. 10, Article ID 105202, 2010.

[7] J. Qian, B. Fang, W. Yang, X. Luan, and H. Nan, "Accurate tilt sensing with linear model," IEEE Sensors Journal, vol. 11, no. 10, pp. 2301-2309, 2011.

[8] J. Yang, W. Wu, Y. Wu, and J. Lian, "Improved iterative calibration for triaxial accelerometers based on the optimal observation," Sensors, vol. 12, no. 6, pp. 8157-8175, 2012.

[9] W. T. Ang, P. K. Khosla, and C. N. Riviere, "Nonlinear regression model of a low-g MEMS accelerometer," IEEE Sensors Journal, vol. 7, no. 1, pp. 81-87, 2007.

[10] S.-H. P. Won and F. Golnaraghi, "A triaxial accelerometer calibration method using a mathematical model," IEEE Transactions on Instrumentation and Measurement, vol. 59, no. 8, pp. 2144-2153, 2010.

[11] H. Zhang, Y. Wu, M. Wu, and Y. Zha, "A multi-position calibration algorithm for inertial measurement units," in Proceedings of the AIAA Guidance, Navigation and Control Conference and Exhibit, AIAA 2008-7437, Honolulu, Hawaii, USA, August 2008.

[12] P. Aggarwal, Z. Syed, and X. Niu, "Cost-effective testing and calibration of low cost mems sensors for integrated positioning, navigation and mapping systems," in Proceedings of the XXIII FIG Congress, pp. 8-13, Munich, Germany, October 2006.

[13] P. Aggarwal, Z. Syed, and N. El-Sheimy, "Thermal calibration of low cost MEMS sensors for land vehicle navigation system," in Proceedings of the IEEE 67th Vehicular Technology Conference, pp. 2859-2863, Singapore, May 2008.

[14] K.-J. Han, C.-K. Sung, and M.-J. Yu, "Improved calibration method for SDINS considering body-frame drift," International Journal of Control, Automation and Systems, vol. 9, no. 3, pp. 497-505, 2011.

[15] H. Zhang, Y. Wu, W. Wu, M. Wu, and X. Hu, "Improved multiposition calibration for inertial measurement units," Measurement Science and Technology, vol. 21, no. 1, Article ID 015107, 2010.

[16] Y. Ren, Y. Wang, M. Wang, S. Wu, and B. Wei, "A measuring system for well logging attitude and a method of sensor calibration," Sensors, vol. 14, no. 5, pp. 9256-9270, 2014.

[17] T. Kariya and H. Kurata, Generalized Least Squares, John Wiley \& Sons, 2004.

[18] S. Łuczak, "Experimental studies of hysteresis in MEMS accelerometers: a commentary," IEEE Sensors Journal, vol. 15, no. 6, pp. 3492-3499, 2015.

[19] W. Yang, B. Fang, Y. Y. Tang, J. Qian, X. Qin, and W. Yao, "A robust inclinometer system with accurate calibration of tilt and azimuth angles," IEEE Sensors Journal, vol. 13, no. 6, pp. 23132321, 2013.

[20] I. Frosio, F. Pedersini, and N. A. Borghese, "Autocalibration of MEMS accelerometers," IEEE Transactions on Instrumentation and Measurement, vol. 58, no. 6, pp. 2034-2041, 2009.

[21] I. Frosio, F. Pedersini, and N. A. Borghese, "Autocalibration of triaxial MEMS accelerometers with automatic sensor model selection," IEEE Sensors Journal, vol. 12, no. 6, pp. 2100-2108, 2012. 


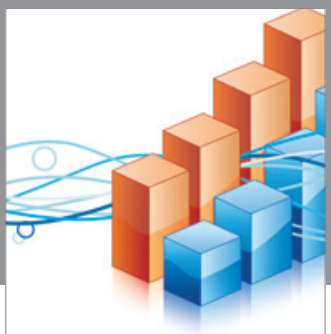

Advances in

Operations Research

vatem alat4

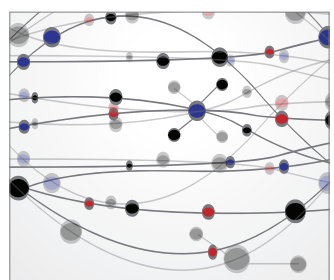

\section{The Scientific} World Journal
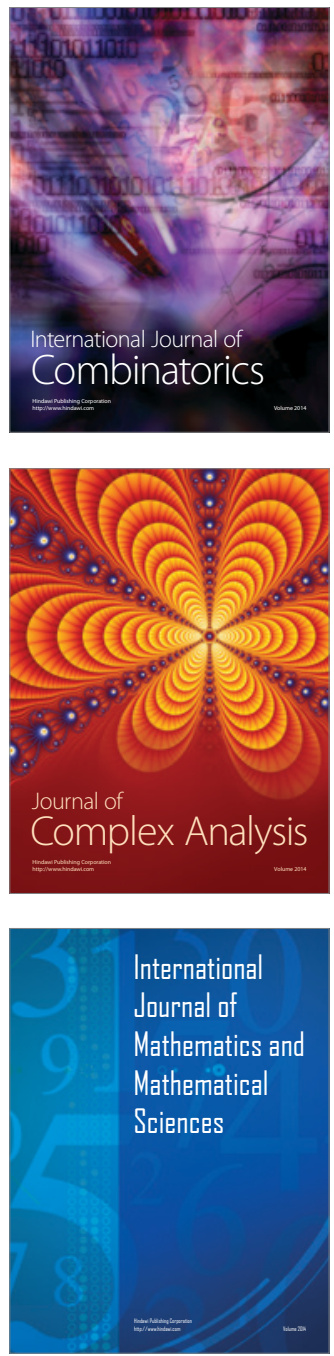
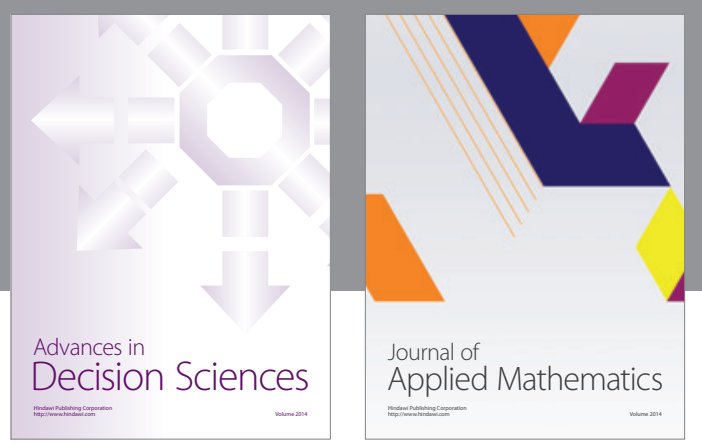

Algebra

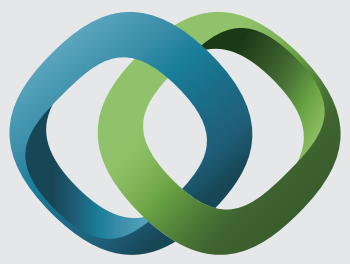

\section{Hindawi}

Submit your manuscripts at

http://www.hindawi.com
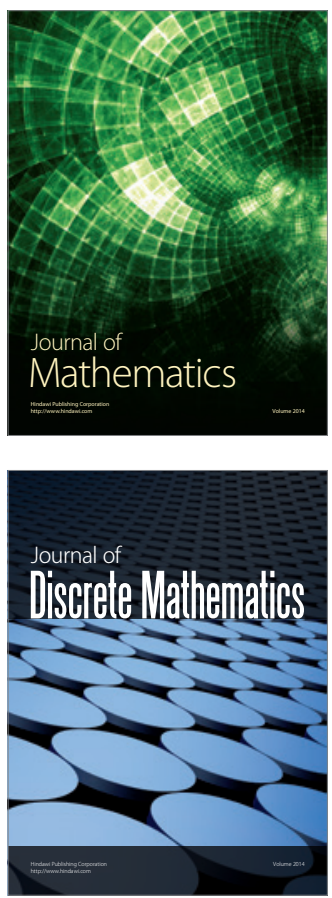

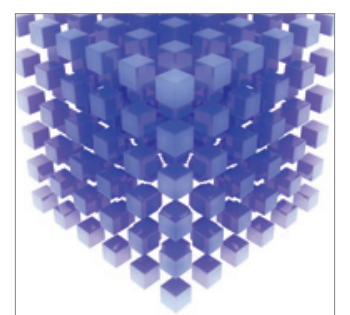

Mathematical Problems in Engineering
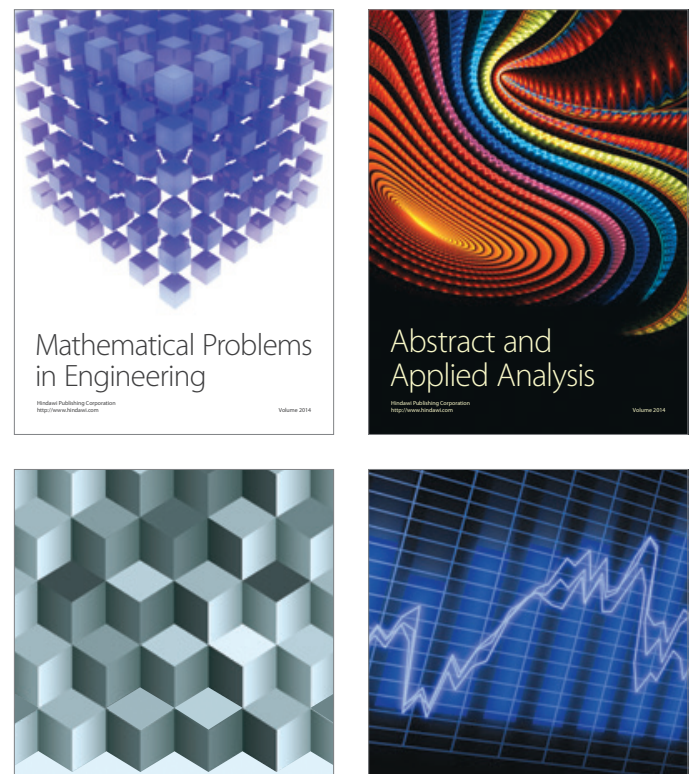

Journal of

Function Spaces

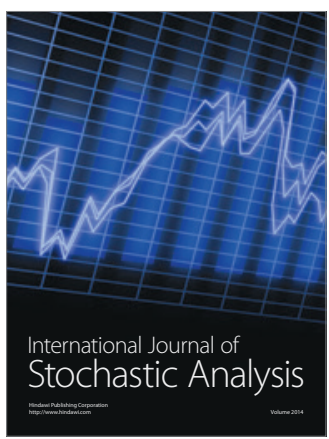

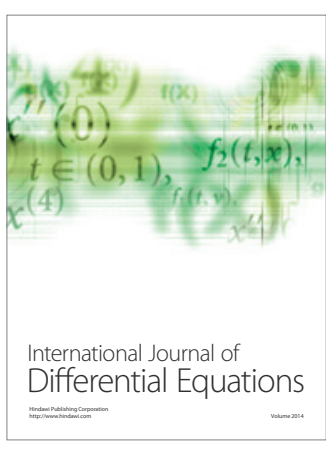
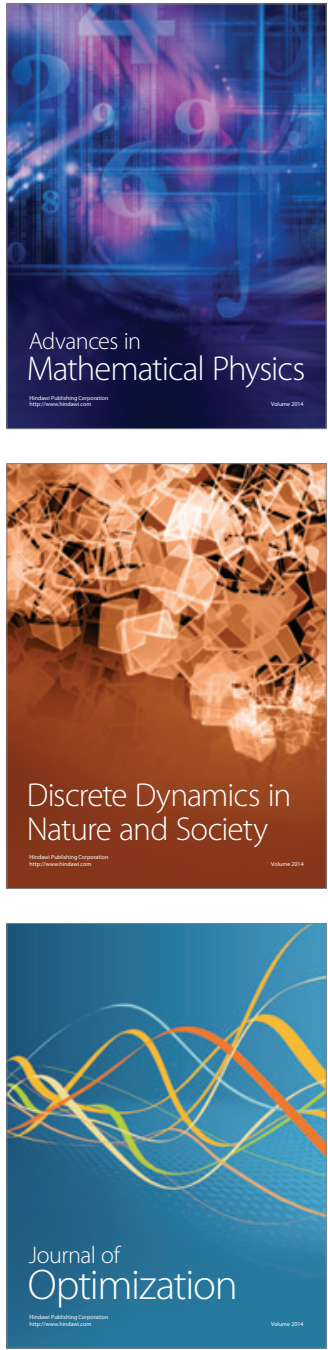